\title{
E. Lazzeri, A. Signore, P.A. Erba, N. Prandini, A. Versari, G. D’Errico, G. Mariani (Editors). Radionuclide Imaging of Infection and Inflammation. A Pictorial Case-Based Atlas. Second Edition
}

\author{
Springer Nature Switzerland AG 2021. ISBN 978-3-030-62174-2
}

\author{
Milena Masone ${ }^{1} \cdot$ Luigi Mansi ${ }^{2}$ \\ Published online: 7 July 2021 \\ (๑) The Author(s), under exclusive licence to Springer-Verlag GmbH Germany, part of Springer Nature 2021
}

The Atlas explores the latest advances in radionuclide imaging of infection and inflammation, specifically illustrating cases obtained with planar and hybrid imaging, with main reference to PET/CT and SPECT/CT. The aim is to provide the reader with the knowledge and clinical interpretation of the most important nuclear medicine techniques, applied either to the diagnosis or monitoring of the pathological processes.

This publication is the second edition of a successful book published by some of the major Italian experts in the field, widely known at the international level. Elena Lazzeri, Paola Anna Erba and Giuliano Mariani work and teach at the Regional Center of Nuclear Medicine, University Hospital of Pisa; Alberto Signore is a professor at the Sapienza University of Rome; Napoleone Prandini is the head of the Nuclear Medicine Department at the Azienda OspedalieroUniversitaria in Modena; Annibale Versari is the director of Nuclear Medicine at the Azienda Unità Sanitaria Locale, IRCCS, in Reggio Emilia; and Giovanni D'Errico works in the Nuclear Medicine Department at the Private Hospital "PIO XI", in Rome.

After the success of the previous edition, published in 2013, widely appreciated by the clinical and scientific community, the Authors propose here an update and integration of the contents. In the last decade, we are witnessing a change in our knowledge of the pathogenesis of inflammation and infection, as well as of the mechanisms that regulate the response to therapy. In clinical practice, we find

Luigi Mansi

Mansi.luigi@libero.it

Ospedale del Mare, Naples, Italy

2 Inter-University Research Center for Sustainability (CIRPS), Rome, Italy ourselves observing different imaging patterns, also due to the change of pathogens, including emerging pathologies, such as those related to the COVID-19 virus. Furthermore, a great technological progress, mainly based on a wider diffusion of more performing hybrid scanners, occurred. With respect to the previous edition, images obtained with newer radiotracers proposed in the study of inflammation/infection have also been introduced.

In each chapter, the most commonly observed patterns, as well as anatomical variants, technical pitfalls and some rarer events are described as didactic cases. The editorial structure, updated to the latest information on pathologies and technologies, includes case reports analysed with a large number of images, which are discussed in the light of the most recent international protocols/guidelines, listed in the text.

A major didactic value is in the representation, in each illustrated case, of the pros and cons of the various imaging methods, comparing radionuclide procedures, in terms of sensitivity and specificity, with alternative techniques. The possible implementation achievable in better defining disease activity or in evaluating prognosis and therapeutic response is also analysed. The aim is to allow the acquirement of a rational, appropriate and justified indication of the use of the various procedures. To further increase the didactic value, updated references and take-home tips are also included at the end of each chapter.

Structured in 356 pages, enriched by 303 illustrations, 213 of which in colour, and an index, the book is divided in the following 16 chapters ( 3 more than the first edition): (1) Normal Findings with Different Radiopharmaceuticals, Techniques, Variants, and Pitfalls; (2) Nuclear Medicine Imaging of Soft Tissue Infections; (3) Nuclear Medicine Imaging of Bone and Joint Infection; (4) Radionuclide 
Imaging of Miscellaneous Bone and Joint Conditions; (5) Nuclear Medicine Imaging of Joint Prosthesis Infections and Peripheral Bone Infections; (6) Nuclear Medicine Imaging of Vascular Prosthesis Infections; (7) Nuclear Medicine Imaging of Non-orthopedic or Cardiovascular Implantable Device Infection; (8) Nuclear Medicine Imaging of Infections and Inflammation of Central Nervous System and of the Head and Neck Structures; (9) Infective Endocarditis and Cardiovascular Implantable Electronic Device Infection; (10) Nuclear Medicine Imaging of Fever of Unknown Origin; (11) Nuclear Medicine Imaging of Abdominal Infections and Inflammations; (12) Nuclear Medicine Imaging of Diabetic Foot; (13) Nuclear Medicine Imaging of Lung Infection; (14) Nuclear Medicine Imaging in Chronic Inflammatory Diseases; (15) Radionuclide Imaging of Inflammatory Vascular Diseases: Vasculitis and Atherosclerosis; (16) Radionuclide Imaging of Infection and Inflammation in Pediatrics.

The reader, both younger and the most experienced, has the opportunity to understand or refresh the pathophysiological bases that underlie the inflammation/infection, becoming more confident in the correct interpretation of the images from the illustration of the cases. This approach may help to orientate more easily in the questions posed in the clinical practice.

In conclusion, this atlas allows an excellent training on the use of techniques and in the interpretation of images acquired with radionuclide procedures. It can result very useful not only to nuclear physicians and radiologists, but also to clinicians, contributing to reach a more correct diagnosis and an effective monitoring of disease activity in patients with inflammatory/infectious pathology.

"The captain is the Science, the practice is the soldiers" (Leonardo da Vinci).

Publisher's note Springer Nature remains neutral with regard to jurisdictional claims in published maps and institutional affiliations. 